\title{
Modeling transducer impulse responses for predicting calibrated pressure pulses with the ultrasound simulation program Field II
}

Bæk, David; Jensen, Jørgen Arendt; Willatzen, Morten

Published in:

Acoustical Society of America. Journal

Link to article, DOI:

10.1121/1.3365317

Publication date:

2010

Document Version

Publisher's PDF, also known as Version of record

Link back to DTU Orbit

Citation (APA):

Bæk, D., Jensen, J. A., \& Willatzen, M. (2010). Modeling transducer impulse responses for predicting calibrated pressure pulses with the ultrasound simulation program Field II. Acoustical Society of America. Journal, 127(5), 2825-2835. https://doi.org/10.1121/1.3365317

\section{General rights}

Copyright and moral rights for the publications made accessible in the public portal are retained by the authors and/or other copyright owners and it is a condition of accessing publications that users recognise and abide by the legal requirements associated with these rights.

- Users may download and print one copy of any publication from the public portal for the purpose of private study or research.

- You may not further distribute the material or use it for any profit-making activity or commercial gain

- You may freely distribute the URL identifying the publication in the public portal 


\title{
Modeling transducer impulse responses for predicting calibrated pressure pulses with the ultrasound simulation program Field II
}

\author{
David Bæk ${ }^{\mathrm{a})}$ and Jørgen Arendt Jensen \\ Department of Electrical Engineering, Center for Fast Ultrasound Imaging, Technical University of \\ Denmark, 2800 Kgs. Lyngby, Denmark

\begin{abstract}
Morten Willatzen
Mads Clausen Institute for Product Innovation, University of Southern Denmark, 6400 Sønderborg, Denmark
\end{abstract}

(Received 14 July 2009; revised 18 December 2009; accepted 24 February 2010)

\begin{abstract}
FIELD II is a simulation software capable of predicting the field pressure in front of transducers having any complicated geometry. A calibrated prediction with this program is, however, dependent on an exact voltage-to-surface acceleration impulse response of the transducer. Such impulse response is not calculated by FIELD II. This work investigates the usability of combining a one-dimensional multilayer transducer modeling principle with the FIELD II software. Multilayer here refers to a transducer composed of several material layers. Measurements of pressure and current from Pz27 piezoceramic disks as well as pressure and intensity measurements in front of a 128 element commercial convex medical transducer are compared to the simulations. Results show that the models can predict the pressure from the piezoceramic disks with a root mean square (rms) error of $11.2 \%$ to $36.2 \%$ with a $2 \mathrm{~dB}$ amplitude decrease. The current through the external driving circuits are predicted within $8.6 \%$ to $36 \%$ rms error. Prediction errors of $30 \%$ and in the range of $5.8 \%-19.9 \%$ for the pressure and the intensity, respectively, are found when simulating the commercial transducer. It is concluded that the multilayer transducer model and the FIELD II software in combination give good agreement with measurements.
\end{abstract}

(C) 2010 Acoustical Society of America. [DOI: 10.1121/1.3365317]

PACS number(s): 43.38.Fx, 43.20.Px, 43.58.Vb [AJZ]

Pages: 2825-2835

\section{INTRODUCTION}

The FIELD II program ${ }^{1,2}$ is widely used within several ultrasound imaging research areas. It is suitable for simulating rf-data for signal processing and testing of several transducer geometries. However, Field II requires knowledge of each of the transducer elements' surface acceleration to perform calibrated pressure predictions. The acceleration is most often measured or approximated by a tapered tone burst. The tapered tone burst method may be found adequate for generating rf-data for signal processing as well as in studies of the transducer geometry where the transducers impulse response is mainly needed for, e.g., a proper matched filtering. Another useful area for the FIELD II software is for intensity simulation by calculating the pressure at a given point of interest. For such simulations to be useful in intensity validation processes, which have to fulfill Food and Drug Administration (FDA) requirements, it is no longer acceptable to have knowledge of an approximated impulse response. Direct measurement of the impulse response may then be needed. Measuring the impulse response is an expensive and cumbersome method, hence a direct simulation of the transducer and its driving circuits could be beneficial. Such an approach would also ease the development of new

\footnotetext{
${ }^{\text {a) }}$ Author to whom correspondence should be addressed. Electronic mail: db@elektro.dtu.dk
}

multilayered transducers and prediction of their pressure field responses.

To simulate the pressure response from transducers, a model which can predict the volt-to-surface acceleration conversion of multilayered transducers is needed. Methods for modeling piezoelectric transducers are well known in literature. ${ }^{3-8}$ Most of these models are based on electrical equivalent circuits benefiting from transmission line theory to represent the electromechanical coupling and acoustic wave propagation, and others rely on deriving impedance matrices for describing the transducer behavior. All of these methods have their advantage and disadvantages depending on the application of use.

This paper, however, investigates the accuracy in combining a one-dimensional modeling principle as described by Willatzen $^{9,10}$ with the FIELD II simulation software. This hybrid modeling principle was briefly discussed in our previous works ${ }^{11,12}$ and is in this work extended and investigated in detail. The model is based on Navier's equations, MaxwellPoisson's law, and piezoelectric constitutive equations and can be easily solved in a closed form using, e.g., Gauss elimination. The modeling principle can account for both electrical and mechanical losses by using the mechanical quality factor and the dielectrical loss factor $\tan (\delta)$ or by using the transducers' characteristic parameters in a complex-valued form which accounts implicitly for attenuation. ${ }^{13}$ Furthermore, by solving the closed form equa- 
tions for a multilayered structure, it is possible to extract knowledge of particle displacement, velocity, acceleration, and dielectrical effects at any position inside a multilayer transducer assumed to operate in its longitudinal mode. The input parameters for the model are those directly found in the constitutive matrices such as the stiffness matrix, the piezoelectric stress constant matrix, and the permittivity matrix wherefore the influence of each constitutive parameter can be studied directly. All these matrices are most often directly available by the material manufacturers and are, thus, not hidden in an effective constant as is the case with electrical equivalent models. Furthermore, the volt-to-surface acceleration impulse response can easily be solved for using the method presented by Willatzen ${ }^{10}$ to any bandwidth of interest. The bandwidth is only limited by the computer power available and may be restricted depending on the number of resonance frequencies needed. The modeling principle is therefore beneficial for both transducer designers and FIELD II users, especially since the modeling principle is mathematically very suitable for predicting this impulse response in a form that can be directly exported for use in the FIELD II software.

In this paper, the modeling principle is used for describing circular Pz27 piezoceramics from Ferroperm Piezoceramics A/S, Kvistgaard, Denmark. The ceramics are mounted with silver electrodes, immersed into water and driven with a function generator. Furthermore, a slightly more complicated transducer model describing a commercial 128 element convex medical ultrasound transducer from BK Medical Aps. (Herlev, Denmark) is presented with a simplified model description of a driving transmitter unit from BK Medical Aps. Pressure predictions along the center axis of the ceramic as well as pressure and intensity predictions in the elevation plane of the convex transducer are performed by combining the models with the FIELD II software. All simulations are compared with measurements to quantify the accuracy of the models.

\section{THEORY}

Section II A describes the basic equations needed to set up a consistent equation set for solving simple resistance loaded piezoceramics as well as more complicated electronic loaded commercial medical transducers. It shows how attenuation and boundary conditions can be included into the transducer model, and it discusses how to set up the FIELD II program for handling the piezoceramics and the convex transducer.

\section{A. Acoustical wave propagation in solid layers}

The time-dependent velocity and tension at any spatial position inside a one-dimensional transducer layer can be found based on monofrequency solutions for the particle velocity $u$ and the tension $T:{ }^{10}$

$$
\begin{aligned}
& u_{33}(z, t, \omega)=\frac{c_{33}^{D} S_{A}}{Z_{a}} e^{(-J k z-J \omega t)}-\frac{c_{33}^{D} S_{B}}{Z_{a}} e^{(J k z-J \omega t)}, \\
& T_{33}(z, t, \omega)=c_{33}^{D}\left[S_{A} e^{(-J k z-J \omega t)}+S_{B} e^{(J k z-J \omega t)}\right]-h_{33} D,
\end{aligned}
$$

where $c_{33}^{D}$ and $h_{33}$ are the stiffness constant in the longitudinal direction and the piezoelectric constant defined as $e_{33} / \epsilon_{33}^{S}$, respectively. Here $e_{33}$ and $\epsilon_{33}^{S}$ are the piezoelectrical coupling coefficient for stress-charge form and the electric permittivity, respectively. The propagation constant $k$ is defined as $\omega / v_{33}$ where $\omega$ is the radial frequency, and $v_{33}$ is the wave velocity defined by $\sqrt{c_{33}^{D} / \rho}$. Here $\rho$ is the given layer's material density. The acoustical impedance $Z_{a}$ is defined as $Z_{a}=v_{33} \rho$. The unknown coefficients $S_{A}$ and $S_{B}$ are frequency and boundary dependent constants, and $D$ is the frequencydependent electric displacement. In the following, any subscripts of 33 are discarded since the transducers are assumed to operate in the longitudinal thickness mode only.

For nonpiezoelectric layers the same solutions are valid. However, the piezoelectrical constant vanishes and the electrical displacement $D$ can be neglected.

Tension and particle velocities must be continuous everywhere and, in particular, at interfaces

$$
\begin{aligned}
& T_{\text {Layer }_{1}}=T_{\text {Layer }_{2}}, \\
& u_{\text {Layer }_{1}}=u_{\text {Layer }_{2}} .
\end{aligned}
$$

\section{B. Modeling losses}

Losses, which influence the transducer response, can mainly be divided into acoustical and electrical losses. ${ }^{10,14-16}$

The acoustical losses arise due to many physical mechanisms such as heating, viscosity, and cross-talks which are all relatively complicated mechanisms to model. Two ways of modeling losses are presented and tested with the modeling here. One method applies purely real-valued transducer parameters from which the attenuation can be approximated explicitly. Another method utilizes a complex-valued transducer parameter set which implicitly accounts for losses. ${ }^{13}$

The real-valued method accounts for losses by using a complex propagation constant $\bar{k}$ instead of a real-valued one in, e.g., Eqs. (1) and (2). A transformation of the real-valued propagation constant may therefore be performed as $k \rightarrow \bar{k}$ $=k^{r}+J \alpha$, where $k^{r}=\omega / v^{r}$ and $v^{r}$ is the phase velocity. The attenuation constant $\alpha$ is an approximation of mechanical losses and has the units $[1 / \mathrm{m}]$. It may be found using the mechanical quality factor, $Q_{m}$, as $\alpha=\pi /\left(Q_{m} \lambda\right)$, where $\lambda$ is the wavelength. A complex phase velocity can therefore be found as

$$
\bar{v}=\frac{\omega}{\bar{k}}=\frac{\omega}{k^{r}+J \alpha}=\frac{v^{r}}{1+\frac{J \alpha v^{r}}{\omega}},
$$

and by substitution the complex propagation constant becomes

$$
\bar{k}=\frac{\omega}{\bar{v}} .
$$

Electrical losses may be defined in two categories: losses due to the electrical network and losses due to the transducer materials. Losses in the electrical network may be very cumbersome to account for since they highly depend on the 


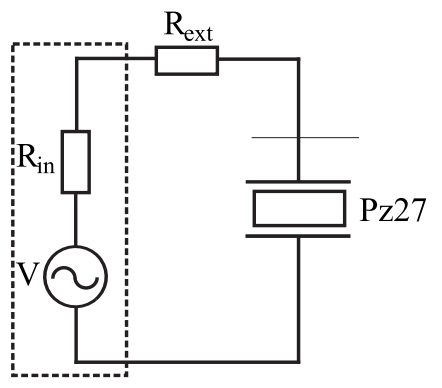

FIG. 1. Schematics of the electronic network loading the Pz27.

transmitter system, cables, element cross-talks, etc. Thorough knowledge of the electrical network design is therefore needed. The dielectrical losses in the transducer, however, can be modeled by defining the dielectrical loss factor as a complex-valued one $\epsilon^{\prime}=\epsilon+J \epsilon \tan \delta$, where $\tan (\delta)$ is the dielectric loss factor. ${ }^{10}$ This method is suitable if only realvalued material constants for the transducer are known.

The second suggested method for modeling both electrical and acoustical losses in the transducer is by applying complex-valued material coefficients for $c^{D}, h, e, \epsilon^{S}$. This method may be theoretically more powerful than the method of using the mechanical quality factor and the dielectric loss factor, since it has been found that loss contributions in piezoelectric materials can be directly included into each material constant when defining them as complex valued. ${ }^{13,17,18}$

\section{Electrical networks}

The circular piezoceramic disks considered in this paper are assumed to be driven with a function generator in series with an internal and an external resistance, $R_{\text {in }}, R_{\text {ext }}$. The schematics is found in Fig. 1. The resistance $R_{\text {ext }}$ seen in the figure is modeled as a series loading resistance. This resistance was used in the practical experiment to determine the current flow through the circuit.

This assumption makes the electrical constitutive equations identical with other works ${ }^{10}$ where the resistance loading is modeled as a simple electrical impedance and gives the following electrical equation to be satisfied:

$$
-J \omega V=-A\left(R_{\mathrm{in}}+R_{\mathrm{ext}}\right) \omega^{2} D-J \omega \frac{L_{p}}{\epsilon^{S}} D-h\left[u\left(L_{p}\right)-u(0)\right],
$$

where $L_{p}$ and $A$ are the piezoelectric thickness and cross section, respectively.

A model for the exact electrical loading of a commercial medical transducer is more complicated to handle. It consists of a coaxial cable and the transmitter unit of the scanner driving the transducer. In this paper, a transmitter from BK Medical Aps. for driving the convex array transducer is applied. A simplified circuit diagram for the applied scanner and transducer is shown in Fig. 2, where the electronic components $L_{1}$ and $L_{2}$ are inductances. $R_{1}, R_{2}$, and $R_{m u x}$ are passive resistances.

The coaxial cable is modeled using lossless transmission line theory where forward and backward traveling voltage and current waves in the frequency domain are given by

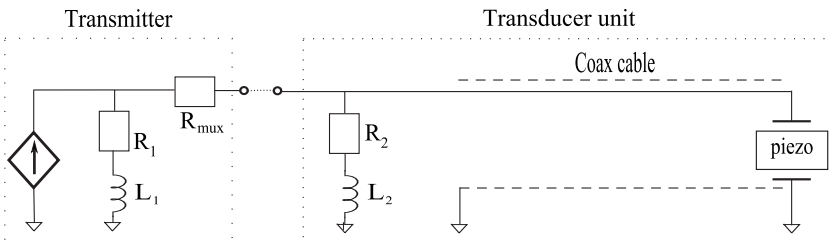

FIG. 2. Simplified electronic network loading the commercial transducer.

$$
\begin{aligned}
& V_{\text {coax }}=V_{+} e^{J \gamma z_{\text {coax }}}+V_{-} e^{-J \gamma z_{\text {coax }},} \\
& I_{\text {coax }}=\frac{V_{+}}{Z_{0}} e^{J \gamma z_{\text {coax }}}-\frac{V_{-}}{Z_{0}} e^{-J \gamma z_{\text {coax }}} .
\end{aligned}
$$

Here $Z_{0}$ is the characteristic impedance of the cable, and $\gamma$ is the propagation constant defined as $\sqrt{L C}$, where $L$ and $C$ are the cable series inductance per unit length and the shunt capacitance per unit length, respectively. The variable $z_{\text {coax }}$ describes the distance along the coaxial cable.

The voltage, $V_{p e}$, across the piezoceramic is defined as

$$
V_{p e}=-J \omega \frac{L_{p}}{\epsilon^{S}} D-h\left[u_{p}\left(L_{p}\right)-u_{p}(0)\right],
$$

where $L_{p}$ is the thickness of the piezoceramic.

The boundary conditions for the coaxial cable at the transducer end have to satisfy both current and voltage continuity. By recalling the relation between electrical displacement and displacement current: $I=A(\partial D / \partial t)$ while having $z_{\text {coax }}=0$ at the transducer end of the cable, the following equations have to be satisfied:

$$
\begin{aligned}
& V_{+}+V_{-}=V_{p e}, \\
& \frac{V_{+}}{Z_{0}}-\frac{V_{-}}{Z_{0}}=-J \omega A D .
\end{aligned}
$$

At the other end of the cable, $z_{\text {coax }}=-L_{\text {coax }}$. Circuit analysis of the cable yields

$$
\begin{aligned}
V(\omega)= & {\left[\frac{Z_{g}}{R_{2}-J \omega L_{2}}+1\right] V_{+} e^{-J \gamma L_{\mathrm{coax}}}+\cdots } \\
& +\left[\frac{Z_{g}}{R_{2}-J \omega L_{2}}-1\right] V_{-} e^{J \gamma L_{\mathrm{coax}}},
\end{aligned}
$$

where $Z_{g}=R_{1}+R_{\text {mux }}-J \omega L_{1}$ (see Fig. 2) and $V(\omega)$ is the Fourier transform of the driving pulse.

\section{Transducer model for the circular piezoceramic disk}

The piezoceramic disks used in testing our model are standard Pz27 piezoceramic samples produced by Ferroperm Piezoceramics A/S, Kvistgård, Denmark. The samples are circular in the cross section and coated with silver electrodes. Measurements were performed submerged into water which gives a setup as shown in Fig. 3. A function generator with an internal and an external series resistance was applied for driving. Therefore, modeling of

$$
T_{\text {back }}=c_{A g}^{D}\left(A_{A g, 1}+B_{A g, 1}\right) \text {, }
$$




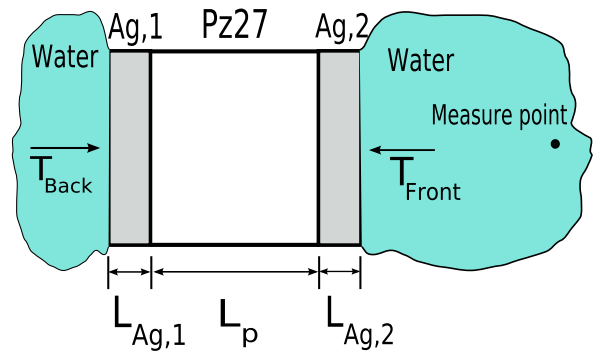

FIG. 3. (Color online) Illustration of the longitudinal cross section of a Pz27 ceramic layered with silver electrodes and submerged into water.

$$
\begin{aligned}
& \frac{T_{\mathrm{back}}}{Z_{\mathrm{back}}}=\frac{c_{A g}^{D}}{Z_{A g}}\left(A_{A g, 1}-B_{A g, 1}\right), \\
& c_{p}^{D}\left(A_{p}+B_{p}\right)-h D=c_{A g}^{D}\left(A_{A g, 1} e^{-J k_{A g} L_{A g}}+B_{A g, 1} e^{J k_{A g} L_{A g}}\right),
\end{aligned}
$$$$
\frac{c_{p}^{D}}{Z_{p}}\left(A_{p}-B_{p}\right)=\frac{c_{A g}^{D}}{Z_{A g}}\left(A_{A g, 1} e^{-J k_{A g} L_{A g}}-B_{A g, 1} e^{J k_{A g} L_{A g}}\right),
$$$$
c_{A g}^{D}\left(A_{A g, 2}+B_{A g, 2}\right)=c_{p}^{D}\left(A_{p} e^{-J k_{p} L_{p}}+B_{p} e^{J k_{p} L_{p}}\right)-h D,
$$$$
\frac{c_{A g}^{D}}{Z_{A g}}\left(A_{A g, 2}-B_{A g, 2}\right)=\frac{c_{p}^{D}}{Z_{p}}\left(A_{p} e^{-J k_{p} L_{p}}-B_{p} e^{J k_{p} L_{p}}\right),
$$$$
T_{\text {front }}=c_{A g}^{D}\left(A_{A g, 2} e^{-J k_{A g} L_{A g}}+B_{A g, 2} e^{J k_{A g} L_{A g}}\right),
$$$$
-\frac{T_{\text {front }}}{Z_{\text {front }}}=\frac{c_{A g}^{D}}{Z_{A g}}\left(A_{A g, 2} e^{-J k_{A g} L_{A g}}-B_{A g, 2} e^{J k_{A g} L_{A g}}\right),
$$$$
-J \omega V(\omega)=-\left(\left(R_{\text {in }}+R_{\text {ext }}\right) \omega^{2} A+J \omega \frac{L_{p}}{\epsilon^{S}}\right) D-h \frac{c_{p}^{D}}{Z_{p}}
$$$$
\times\left(A_{p}-B_{p}\right)+h \frac{c_{A g}^{D}}{Z_{A g}}\left(A_{A g, 2}-B_{A g, 2}\right),
$$

this setup using the above described modeling method, yields a three layer solid transducer model, which is described by Eqs. (14)-(22). These equations model the electronics as resistances in series with a function generator having an internal resistance, $R_{\text {in }}$, and an external series resistance, $R_{\text {ext }}$.

The subscripts $A g_{1}, A g_{2}$, and $p$ in Eqs. (14)-(22) are referring to the first silver layer, the second silver layer, and the piezoceramic layer, respectively, and they are used as subscripts for the unknown coefficients $A$ and $B$. This model involves nine equations with nine unknowns to be solved in MATLAB.

\section{E. Transducer model for a 128 element convex array}

A medical convex array has a more complicated structure than regular circular disks as considered above. It consists of several matching layers, and the surface is geometrically more complicated. However, the modeling of each element's impulse response follows the same principle as discussed above. Each element of the convex transducer under consideration in this paper consists of a backing layer, a
Major blade width:

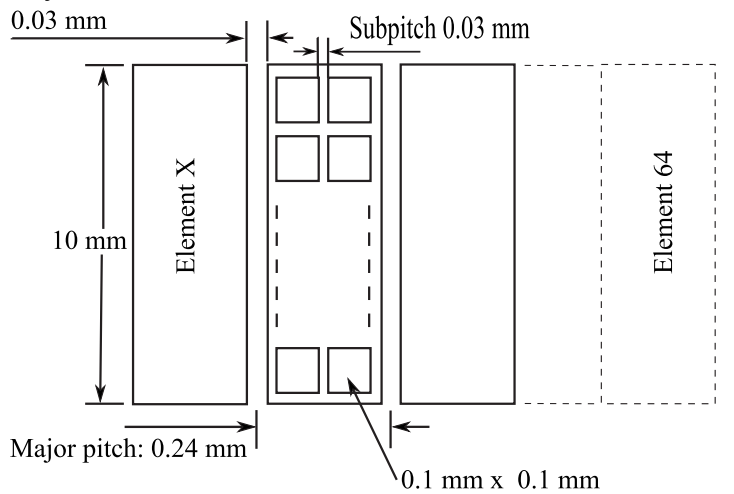

FIG. 4. Illustration of the subdivided elements of a convex ultrasound transducer under consideration.

piezoceramic layer, a first matching layer (ML1), a second matching layer (ML2), and a lens. Each of the 128 major elements consists of smaller subelements which are created as subdivided elements in two columns. Each subelement measures a rectangle of $0.1 \times 0.1 \mathrm{~mm}^{2}$ as shown in Fig. 4 . Together all the subelements and subpitch measure $10 \mathrm{~mm}$ in height and define the major elements of which there are 128. The transducer has a convex radius of $29 \mathrm{~mm}$ and an elevation focus at $70 \mathrm{~mm}$.

Considering the electronic network to be as shown in Fig. 2, and with parameter knowledge of the different layers for the different elements one can establish a consistent set of Eqs. (23)-(35) for this transducer.

$$
\begin{aligned}
& T_{\text {back }}=c_{p}^{D}\left(A_{p}+B_{p}\right)-h D, \\
& \frac{T_{\text {back }}}{Z_{\text {back }}}=\frac{c_{p}^{D}}{Z_{p}}\left(A_{p}-B_{p}\right), \\
& c_{\mathrm{ML} 1}^{D}\left(A_{\mathrm{ML} 1}+B_{\mathrm{ML} 1}\right)=c_{p}^{D}\left(A_{p} e^{-J k_{p} L_{p}}+B_{p} e^{J k_{p} L_{p}}\right)-h D,
\end{aligned}
$$

$$
\frac{c_{\mathrm{ML} 1}^{D}}{Z_{\mathrm{ML} 1}}\left(A_{\mathrm{ML} 1}-B_{\mathrm{ML} 1}\right)=\frac{c_{p}^{D}}{Z_{p}}\left(A_{p} e^{-J k_{p} L_{p}}-B_{p} e^{J k_{p} L_{p}}\right),
$$$$
c_{\mathrm{ML} 2}^{D}\left(A_{\mathrm{ML} 2}+B_{\mathrm{ML} 2}\right)=c_{\mathrm{ML} 1}^{D}\left(A_{\mathrm{ML} 1} e^{-J k_{\mathrm{ML} 1} L_{\mathrm{ML} 1}}\right.
$$$$
+B_{\mathrm{ML} 1} e^{\left.J k_{\mathrm{ML} 1} L_{\mathrm{ML} 1}\right)} \text {, }
$$

$\frac{c_{\mathrm{ML} 2}^{D}}{Z_{\mathrm{ML} 2}}\left(A_{\mathrm{ML} 2}-B_{\mathrm{ML} 2}\right)=\frac{c_{\mathrm{ML} 1}^{D}}{Z_{\mathrm{ML} 1}}\left(A_{\mathrm{ML} 1} e^{-J k_{\mathrm{ML} 1} L_{\mathrm{ML} 1}}\right.$

$$
-B_{\mathrm{ML} 1} e^{\left.J k_{\mathrm{ML} 1} L_{\mathrm{ML} 1}\right)} \text {, }
$$

$$
c_{\text {lens }}^{D}\left(A_{\text {lens }}+B_{\text {lens }}\right)=c_{\mathrm{ML} 2}^{D}\left(A_{\mathrm{ML} 2} e^{-J k_{\mathrm{ML} 2} L_{\mathrm{ML} 2}}\right.
$$$$
+B_{\mathrm{ML} 2} e^{\left.J k_{\mathrm{ML} 2} L_{\mathrm{ML} 2}\right)} \text {, }
$$

$\frac{c_{\text {lens }}^{D}}{Z_{\text {lens }}}\left(A_{\text {lens }}-B_{\text {lens }}\right)=\frac{c_{\mathrm{ML} 2}^{D}}{Z_{\mathrm{ML} 2}}\left(A_{\mathrm{ML} 2} e^{-J k_{\mathrm{ML} 2} L_{\mathrm{ML} 2}}\right.$

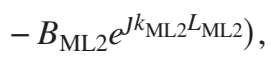

$T_{\text {front }}=c_{\text {lens }}^{D}\left(A_{\text {lens }} e^{-J k_{\text {lens }} L_{\text {lens }}}+B_{\text {lens }} e^{J k_{\text {lens }} L_{\text {lens }}}\right)$, 
TABLE I. Model parameters for Pz27.

\begin{tabular}{lccc}
\hline \hline & Real case & Complex case & \\
\hline$\rho$ & 7700 & 7700 & $\mathrm{~kg} / \mathrm{m}^{3}$ \\
$c_{p}^{D}$ & $1.44 \times 10^{11}$ & {$[(813.9+J 0.113) \pm(0.7-J 0.006)] \times 10^{10}$} & $\mathrm{~N} / \mathrm{m}^{2}$ \\
$h$ & $1.98 \times 10^{9}$ & {$[(21.0+J 0.148) \pm(0.4-J 0.004)] \times 10^{8}$} & $\mathrm{~V} / \mathrm{m}$ \\
$\epsilon^{S}$ & $914 \epsilon_{0}$ & {$[(822-J 10.3) \pm(12-J 0.4)] \times \epsilon_{0}$} & $\mathrm{~F} / \mathrm{m}$ \\
$\tan \delta$ & 0.017 & 0.017 & \\
$\rho_{A g}$ & 10600 & 10600 & $\mathrm{~kg} / \mathrm{m}^{3}$ \\
$c_{A g}^{D}$ & $1.13 \times 10^{11}$ & $1.13 \times 10^{11}$ & $\mathrm{~N} / \mathrm{m}^{2}$ \\
$\rho_{w}$ & 1000 & 1000 & $\mathrm{~kg} / \mathrm{m}^{3}$ \\
$c_{w}^{D}$ & $2.19 \times 10^{9}$ & $2.19 \times 10^{9}$ & $\mathrm{~N} / \mathrm{m}^{2}$ \\
\hline \hline
\end{tabular}

$$
\begin{aligned}
& -\frac{T_{\text {front }}}{Z_{\text {front }}}=\frac{c_{\text {lens }}^{D}}{Z_{\text {lens }}}\left(A_{\text {lens }} e^{-J k_{\text {lens }} L_{\text {lens }}}-B_{\text {lens }} e^{\left.J k_{\text {lens }} L_{\text {lens }}\right)}\right. \\
& -J \omega V_{+}+-J \omega V_{-}=-J \omega \frac{L_{p}}{\epsilon} D-\left[h \frac{c_{\mathrm{ML} 1}^{D}}{Z_{\mathrm{ML} 1}}\left(A_{\mathrm{ML} 1}-B_{\mathrm{ML} 1}\right)\right. \\
& -J \omega A D=\frac{1}{Z_{0}} V_{+}-\frac{1}{Z_{0}} V_{-} \\
& \left.\quad-h \frac{c_{p}^{D}}{Z_{p}}\left(A_{p}-B_{p}\right)\right] \\
& V(\omega)=\left(\frac{Z_{g}}{Z_{0}}+\frac{Z_{g}}{R_{2}-J \omega L_{2}}+1\right) V_{+} e^{J \gamma L_{\mathrm{coax}}} \\
& +\left(-\frac{Z_{g}}{Z_{0}}+\frac{Z_{g}}{R_{2}-J \omega L_{2}}+1\right) V_{-} e^{-J \gamma L_{\mathrm{coax}}}
\end{aligned}
$$

\section{NUMERICAL SIMULATION}

\section{A. Solving the equations}

The equation sets (14)-(35) are solved in MATLAB for each frequency component at a time for the bandwidth of interest. The surface acceleration is found from Eq. (1) evaluated at the interface between water and the front layer (the lens). It is then multiplied with $-J \omega$ to convert from velocity to acceleration, before the inverse Fourier transform

$$
f(t)=\frac{1}{\sqrt{2 \pi}} \int_{-\infty}^{\infty} F(\omega) e^{-J \omega t} d t .
$$

is used for calculating the resulting impulse response.

\section{Model data for Pz27 ceramics}

The model input data set used for investigation with Pz27 materials is found in Table I. The table contains two sets of data. One set is a real-valued data set of material constants for testing of the model with attenuation based on the mechanical quality factor and the dielectrical loss factor. This set was manufacturer supplied. A second set consists of complex-valued data of material constants measured by Algueró et al. ${ }^{13}$ These data are in their work supplied with a standard deviation which is the added/subtracted terms in the Table I. However, our analysis shows that best amplitude results are found by setting the deviation to zero for $h$ and $\epsilon^{S}$
TABLE II. Simulation parameters for the convex ultrasound transducer.

\begin{tabular}{lcc}
\hline & Value & Unit \\
\hline$\rho_{p}$ & 7870 & $\mathrm{~kg} / \mathrm{m}^{3}$ \\
$\rho_{\mathrm{ML} 1}$ & 2140 & $\mathrm{~kg} / \mathrm{m}^{3}$ \\
$\rho_{\mathrm{ML} 2}$ & 1130 & $\mathrm{~kg} / \mathrm{m}^{3}$ \\
$\rho_{\text {lens }}$ & 1260 & $\mathrm{~kg} / \mathrm{m}^{3}$ \\
$\rho_{\text {back }}$ & 2160 & $\mathrm{~kg} / \mathrm{m}^{3}$ \\
$\rho_{\text {front }}$ & 1000 & $\mathrm{~kg} / \mathrm{m}^{3}$ \\
$c_{p}^{D}$ & $1.103 \times 10^{11}$ & $\mathrm{~N} / \mathrm{m}^{2}$ \\
$c_{\mathrm{ML} 1}^{D}$ & $1.750 \times 10^{10}$ & $\mathrm{~N} / \mathrm{m}^{2}$ \\
$c_{\mathrm{ML} 2}^{D}$ & $4.983 \times 10^{9}$ & $\mathrm{~N} / \mathrm{m}^{2}$ \\
$c_{\text {lens }}^{D}$ & $1.235 \times 10^{9}$ & $\mathrm{~N} / \mathrm{m}^{2}$ \\
$c_{\text {back }}^{D}$ & $1.017 \times 10^{10}$ & $\mathrm{~N} / \mathrm{m}^{2}$ \\
$c_{\text {front }}^{D}$ & $2.372 \times 10^{9}$ & $\mathrm{~N} / \mathrm{m}^{2}$ \\
$\epsilon^{S}$ & $11.5 \times 10^{-9}$ & $\mathrm{~F} / \mathrm{m}$ \\
$h$ & $1.713 \times 10^{9}$ & $\mathrm{~V} / \mathrm{m}^{2}$ \\
$\tan (\delta)$ & 0.16 & \\
\hline \hline
\end{tabular}

and $+(0.7-J 0.006) \times 10^{10}$ for the stiffness constant $c_{p}^{D}$ which is the maximum allowable value according to Algueró et $a l .{ }^{13}$ The complex-valued data set is suitable for testing how well the model works with complex input data. The density of both the real-valued and the complex-valued data set is lowered with $2.5 \%$, which is within the manufacturers tolerances. The small adjustments is made to create the best amplitude pulse shape for long pulses. This also gives the best shape for short pulses.

Two thicknesses of the piezoceramics were applied: 0.94 and $0.5 \mathrm{~mm}$ corresponding to a resonance frequency of 2.1 $\mathrm{MHz}$ and 4.0 MHz, respectively. Both types of ceramics were coated with silver with an estimated thickness of $9 \mu \mathrm{m}$. The ceramics were driven with a function generator having an internal resistance $R_{\text {in }}$ of $50 \Omega$ and an external resistance $R_{\text {ext }}$ of $47.5 \Omega$. The radius of the ceramics was estimated to be $5.05 \mathrm{~mm}$ and the attenuation constants were calculated to $\alpha_{2.1 \mathrm{MHz}}=19 \mathrm{~m}^{-1}$ and $\alpha_{4 \mathrm{MHz}}=36 \mathrm{~m}^{-1}$ for the 2.1 $\mathrm{MHz}$ samples and the 4.0 $\mathrm{MHz}$ samples, respectively.

\section{Model data for the convex transducer}

Table II lists the input parameters needed for predicting the impulse response of the convex ultrasound transducer. The thicknesses of the different layers $L_{p}, L_{\mathrm{ML} 1}, L_{\mathrm{ML} 2}$, and $L_{\text {lens }}$ are set to $0.38,0.17,0.11$, and $0.46 \mathrm{~mm}$, respectively. The attenuation coefficients are calculated to be $\{1036,0,139,186,308,0.025\}[1 / \mathrm{m}]$ for the backing layer, the piezoceramic layer, the ML1, the ML2, the lens, and water, respectively.

The electronic components $R_{1}, R_{2}, R_{\text {mux }}, L_{1}$, and $L_{2}$ found in Fig. 2 are set to $100 \Omega, 20 \Omega, 22 \Omega, 3.9 \mu \mathrm{H}$, and $10 \mu \mathrm{H}$, respectively. The coaxial cable has a characteristic impedance of $75 \Omega$, a length $L_{\text {coax }}$ of $2.2 \mathrm{~m}$, an estimated inductance per unit length, $L$, of $0.387 \mu \mathrm{H} / \mathrm{m}$, and an estimated capacitance per unit length, $C$, of $67 \mathrm{pF} / \mathrm{m}$. The later estimations are taken from product descriptions of similar coaxial cables. This estimation is found acceptable through simulation investigations on the influence of these parameters for the final results. This was done by varying the parameters and identifying the effect on the pressure pulse. 
This showed that an exact value does not have significant influence on the errors. To provide an estimate of the input waveform for the FIELD II model, the transmitter output was measured without the transducer being present.

\section{B. Setting up FIELD II}

For FIELD II to calculate the pressure, the surface acceleration and the transducer surface geometry must be defined. For the piezoceramic disks FIELD II was set up to calculate the point pressure field using square element sizes of 0.1 $\times 0.1 \mathrm{~mm}^{2}$ for the piston model.

For the convex array two possible surface models with which to set up FIELD II were investigated. One setup models each single subelement on the surface of the lens. In reality the lens material has a continuous smooth surface without any subdicing hence waves propagating from the underlying subdiced materials through the lens will propagate spherically forward toward the lens surface. These waves will superpose at the front surface according to Huygen's principle and give an approximate plane wave transmission. Modeling of each single element therefore assumes no superposition in the lens material.

The second setup also assumes one-dimensional operation of the lens surface. Here, however, the operation of each major element is modeled as vibrating in one dimension only and as if no dicing occurs on the underlying materials. This defines the area, $A$, in Eq. (34) to be the area of the total major element, whereas for the first setup $A$ is the size of each subdivided element as shown in Fig. 4.

FIELD II supports two possible built-in functions for modeling the convex transducer, $x d c \_c o n v e x \_f o c u s e d \_m u l t i-$ row, and $x d c \_c o n v e x \_f o c u s e d \_a r r a y$. The first function is suitable for setting up each subelement, and the second is suitable for modeling only the major elements.

The pressure at the points of interest along the centerline of an active element was calculated by simulation. At each measurement point in front of the convex transducer, the pressure at several points within the radius of the needle hydrophone was calculated. These point pressures were then used to account for the hydrophone area by averaging over the surface:

$$
p_{\text {hyd }}=\frac{1}{A_{\text {hyd }}} \int_{A_{\text {hyd }}} p(\bar{r}, t) d S,
$$

where $p_{\text {hyd }}$ is the average pressure detected by the hydrophone, $p(\bar{r}, t)$ is the spatial pressure calculation by FIELD II, and $A_{\text {hyd }}$ is the area of the hydrophone. This integral is solved numerically as

$$
p_{\text {hyd }}=\frac{1}{\sum_{i=0}^{N} \Delta s_{i}} \sum_{i=0}^{N} p_{i}(\bar{r}, t) \Delta s_{i},
$$

where $N$ is the number of integration areas and $p_{i}$ is the calculated pressure at the center of the area $\Delta s_{i}$.

The sampling frequency for the convex array modeling was set to $400 \mathrm{MHz}$. The pressure were calculated using 40 times 40 mathematical elements on the major elements.

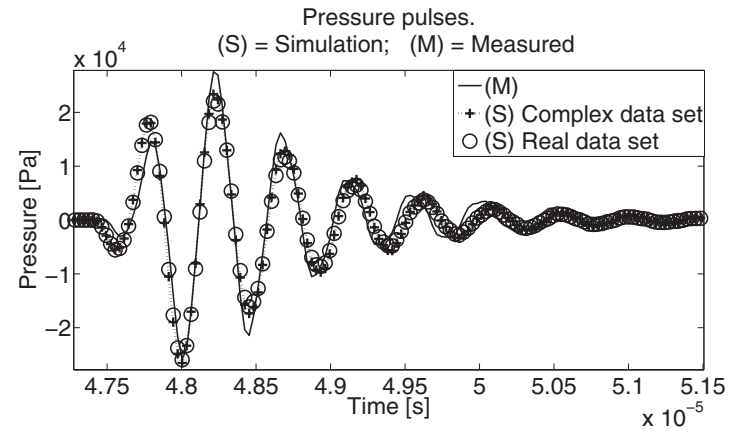

FIG. 5. Pressure comparison between measured (solid) and simulated pressure with a $2.1 \mathrm{MHz}$ driving frequency. Two simulation parameter sets were applied. One complex-valued (dotted line+cross) and one real-valued (circles).

\section{MEASUREMENTS}

\section{A. The Pz27 ceramic disks}

The ceramic disks were driven by an Agilent 33220A function generator that supplied a tone burst of 1,4 , and 10 cycles to the samples with a $20 \mathrm{Vpp}$ from the internal source $V$ in Fig. 1. The pressure pulses were captured with a needle hydrophone having a $250 \mu \mathrm{m}$ radius and were sampled using a remote controlled Agilent MSO6014A oscilloscope. The oscilloscope was set up to average 128 times to lower the noise level, which was found relatively high for the samples. Measurements were performed on five different samples at $2.1 \mathrm{MHz}$ and on five different samples at 4.0 MHz. Measurements were performed submerged into demineralized water, and the hydrophone was each time centered along the center axis of the elements to find the peak amplitude.

\section{B. The convex array}

Measurements with this transducer were performed on three center elements in three depths, 33, 72, and $112 \mathrm{~mm}$ along each of the element's centerlines. A shooting sequence was applied where only the considered element transmitted. Furthermore, at each of the three depths the pressures in the elevation plane were measured. The transducer was driven at 4.0 MHz using a BK Medical Aps. transmitter, delivering a 10 cycle tone burst with a limited peak amplitude of $31 \mathrm{~V}$. The output voltage was limited to avoid too high nonlinear effects on the pressure pulses. All these setups were achieved by using our in house RASMUS (Ref. 19) system, which can be used to drive a transducer in transmit and receive with arbitrary configuration.

\section{RESULTS}

\section{A. The results on the ceramic disks}

Five transducer samples with a resonance frequency of 2.1 $\mathrm{MHz}$ and five transducer samples with a resonance frequency of $4.0 \mathrm{MHz}$ have been measured. The average of these samples are used as a reference for comparison in the following. This has been done to minimize measurement uncertainties as well as differences in the samples.

Figure 5 shows a comparison between measured and 


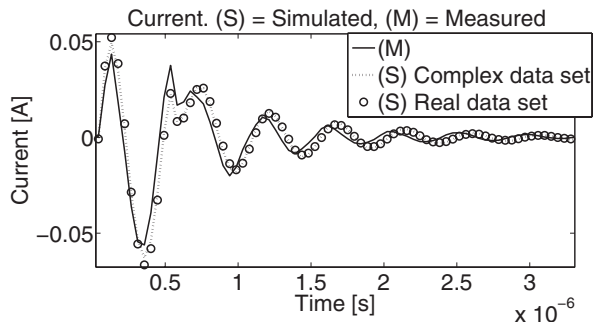

FIG. 6. Current comparison between measured (solid) and simulated pressure through the electronic circuit. Two simulation parameter sets were applied. One complex-valued (dotted line). One real-valued (circle).

simulated pressure pulses. The measured pulse is compared to simulations using the complex-valued and real-valued data sets. The comparison is made with an excitation pulse of 1 cycle at $2.1 \mathrm{MHz}$. Results indicate good agreement between measurement and model output which is a general characteristic for all combinations of pulses and samples that have been tested. A short pulse of 1 cycle has been chosen in Fig. 5 because a one-pulse excitation is the most difficult excitation situation to capture for the model. From the figure a slight overshoot is found as well as a slight tail drifting. From consecutive measurements it has been found that an amplitude decrease of the prediction of around $2 \mathrm{~dB}$ is needed to achieve the lowest error calculation. This amplitude adjustment has been used in the following results.

An example of current comparison between measured and simulated current flow through the driving circuit of the piezoceramic disk setup is shown in Fig. 6. This figure clearly exemplifies that the current is captured very well. A 1 cycle excitation of $2.1 \mathrm{MHz}$ was applied here as well and it can be identified that the pulse tail exhibits the same drifting behavior as the above pressure pulse. Furthermore, it is noticeable that the model captures the rapid change in pulse behavior found in the beginning of the oscillating tail, indicating that the model has a certain capability to capture sudden changes in the pulse behavior.

Table III shows the results of a quantitative error calculation of the model's accuracy compared to the mean of the measurement as well as the deviation in the physical samples themselves. The sample deviations are calculated as the root mean square (rms) error relative to the rms of the mean of the measured pulses and is for a given measurement sequence defined as

$$
\mathrm{rms}_{m}=100 \cdot \frac{\sqrt{\frac{1}{N_{m}} \sum_{m=1}^{N} \frac{1}{N_{s} \sum_{i=1}^{N_{s}}(\bar{P}(m)-P(m, i))^{2}}}}{\sqrt{\frac{1}{N_{m=1}^{N} \bar{P}(m)^{2}}}} .
$$

Here $\bar{P}(m), P(m, i)$, and $N$ are the mean pressure of $N_{s}$ piezoceramic samples, the measured pressure for the $i$ th ceramic sample, and the number of time samples acquired, respectively.

The error of the simulation is calculated as the relative error with respect to the mean of the measurement. This is calculated as

$$
\mathrm{rms}_{s}=100 \cdot \frac{\sqrt{\frac{1}{N} \sum_{m=1}^{N}\left[\left(\bar{P}(m)-P_{s}(m)\right)^{2}\right]}}{\sqrt{\frac{1}{N} \sum_{m=1}^{N} \bar{P}(m)^{2}}},
$$

where $P_{s}$ is the pressure predicted by the model. From Table III it can be identified that the model has a significantly higher rms error in all predictions relative to the average of the measurements than each sample deviates from the average. This is a consequence of the drifting tail behavior and the slight overshoot on the transitions as is indicated on Fig. 5. The errors may seem high, however, it must be recalled that the rms error calculation is a very sensitive method of quantification. Therefore, a slight drift in phase results in a high error contribution.

From the table it becomes clear that the measured current standard deviation is ranging from $3.2 \%$ to $12.1 \%$ for short pulses and from $3.7 \%$ to $7.7 \%$ for long pulses, where best behavior is found for the $2.1 \mathrm{MHz}$ pulses. For pressure measurements it is found that short pulses can be measured with a deviation error around $26 \%$ down to $8.5 \%$, where best results are found for long pulses with a center frequency of 2.1 MHz. The error on the current simulation is found to be higher than the measurement deviation. The maximum simulated current error is found to be around $36.0 \%$ for short pulses while for long pulses the error is $17.9 \%$. The best current result is found in the $4.0 \mathrm{MHz}$ simulation, where a $8.6 \%$ error is found. Comparison of the current error calculated relative to simulations using either the real-valued data set or the complex-valued data set is seen to exhibit almost identical error prediction relative to the measured. This indi-

TABLE III. rms error in percentages for the model and measurements relative to the mean of the measurements using 1,4 , and 10 cycle excitations at 2.1 and 4.0 MHz resonance frequencies, respectively. Current $(I)$ and pressure $(P)$ errors are shown. Errors using either a real-valued data set $(\mathrm{Re})$ or a complex-valued

\begin{tabular}{|c|c|c|c|c|c|c|c|c|c|}
\hline & \multicolumn{3}{|c|}{1 cycle excitation } & \multicolumn{3}{|c|}{4 cycle excitation } & \multicolumn{3}{|c|}{10 cycle excitation } \\
\hline & $\operatorname{Re}$ & C & $M$ & $\operatorname{Re}$ & C & $M$ & $\operatorname{Re}$ & $C$ & $M$ \\
\hline I: $2.1 \mathrm{MHz}$ & 36.0 & 35.5 & 3.2 & 24.9 & 25.4 & 4.8 & 17.1 & 17.9 & 3.7 \\
\hline I: $4 \mathrm{MHz}$ & 29.0 & 25.3 & 12.1 & 16.0 & 14.3 & 9.9 & 9.8 & 8.6 & 7.7 \\
\hline$P: 2.1 \mathrm{MHz}$ & 36.2 & 36.0 & 10.0 & 21.6 & 24.4 & 8.5 & 14.3 & 20.0 & 8.5 \\
\hline$P: 4 \mathrm{MHz}$ & 20.3 & 25.2 & 25.3 & 13.7 & 18.2 & 24.8 & 11.2 & 18.4 & 25.8 \\
\hline
\end{tabular}
data set $(C)$ in the simulation are shown. It is compared against deviations in the measurements $(M)$. 


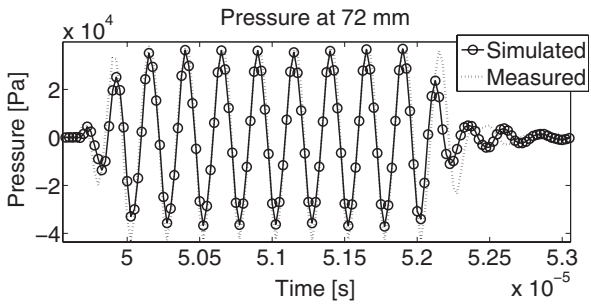

FIG. 7. Comparison between simulated and measured pressure pulses at a distance of $72 \mathrm{~mm}$ from a single element modeling each subdivided element.

cates that the model is capable of working with both types of data set and that the attenuation based on the mechanical quality factor is capturing the acoustic losses with a maximum difference of $3.7 \%$. Prediction errors for the pressure pulses are found to range from $11.2 \%$ to $36.2 \%$ rms error with best results found with the $4.0 \mathrm{MHz}$ center frequency. The 4.0 MHz predicted pulses are found to be closer to the mean measurement than each individual piezoceramic sample. This indicates the importance of using several samples for comparison, which gives the level of accuracy that one can expect.

\section{B. Results for the convex transducer when modeling each single subelement}

A comparison between the simulated and the measured pressure at a distance of $72 \mathrm{~mm}$ along the center axis of an active element is shown in Fig. 7. Several measurements and tests in different depths have shown that the simulations undershoot the amplitude by approximately $1.7 \mathrm{~dB}$ relative to the mean measurement, which is the value found to give the smallest rms error. The following rms results are therefore calculated with this adjustment in amplitude. From Fig. 7 it is furthermore found that the model captures the pulse behavior relatively well. Only a slight overshoot on the transition is found and some missing behavior on the pulse tail can be seen.

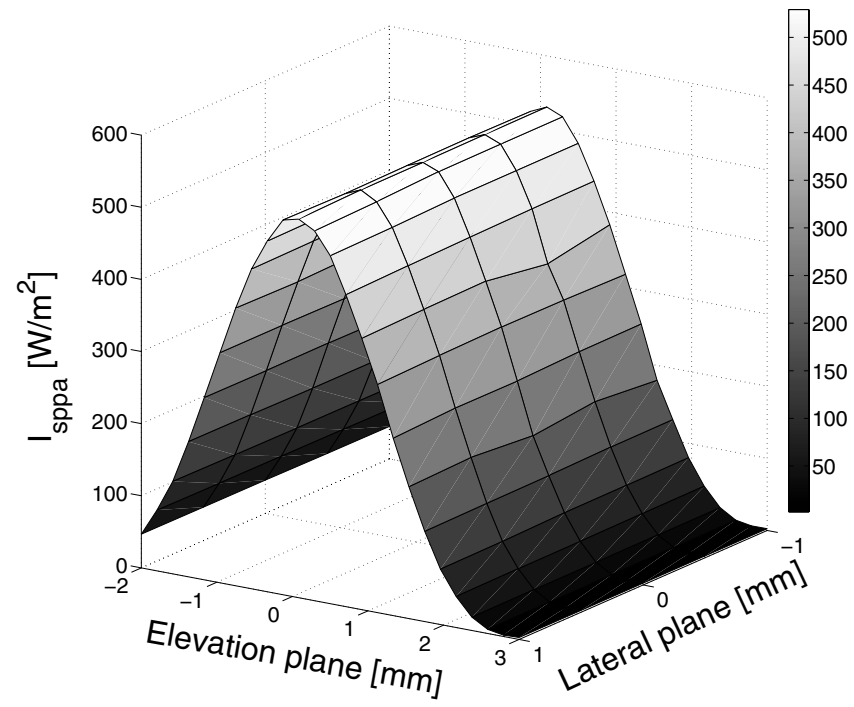

FIG. 8. Intensity profile measured at a $72 \mathrm{~mm}$ distance from an active element in the lateral plane and the elevation plane. The colors also indicate the intensity level.
TABLE IV. Intensity profiles' relative rms errors measured at three depths.

\begin{tabular}{lccc}
\hline \hline & \multicolumn{3}{c}{ rms \% intensity } \\
\hline Depth (mm) & 33 & 72 & 112 \\
Element 64 & 3.4 & 8.9 & 0.9 \\
Element 65 & 1.0 & 0.6 & 1.5 \\
Element 66 & 1.5 & 1.1 & 1.1 \\
\hline
\end{tabular}

Figure 8 is an example of a measured intensity profile at a distance of $72 \mathrm{~mm}$ from an active element. The profile is measured in the elevation and the lateral direction and is calculated as the spatial peak pulse average intensity, which is chosen due to its suitability for pulse comparison. When comparing a simulated intensity profile in the elevation plane with measurements, it is very important that the proper lateral direction is found in the measurement. If this is not found one may compare with a wrong measurement profile. As is seen in Fig. 8 the deviation of the intensity profiles in the lateral direction can be seen to be small and may be quantified by an rms error calculated as

$$
\mathrm{rms}_{\mathrm{prof}}=100 \cdot \frac{\sqrt{\frac{1}{N_{m=1}^{N}} \sum_{\frac{1}{N_{s}} \sum_{i=1}^{N_{s}}(\bar{I}(m)-I(m, i))^{2}}}}{\sqrt{\frac{1}{N_{m=1}^{N}} \sum_{m}^{N} \bar{I}(m)^{2}}},
$$

where $\bar{I}(m), I(m, i), N_{s}$, and $N$ are the mean intensity profile, the measured intensity profile at the $i$ th lateral direction, the number of lateral profiles, and the number of elevation points measured, respectively. Table IV lists calculated rms errors for measurements of intensity profiles from three center elements at three different depths: 33, 72, and $112 \mathrm{~mm}$.

Table IV shows that measurements on Element 65 and 66 are within a $1.5 \%$ deviation relative to the mean but increasing for Element 64 around the focus point. Therefore, a simulated intensity profile compared with an intensity profile for Element 65 and 66 for any of the lateral directions can be made with similar error predictions. However, Element 64 may give a highly different error prediction due to the high relative deviation of its measured lateral profiles. Therefore, in the following, all simulated intensity profiles along the elements' center axes are compared with the laterally average of measured intensity profiles.

Figure 9 shows an intensity comparison in the elevation plane between simulated and averaged intensity profiles at the three depths. From the results plotted in the figure, it is found that the tendency of the intensity level is captured

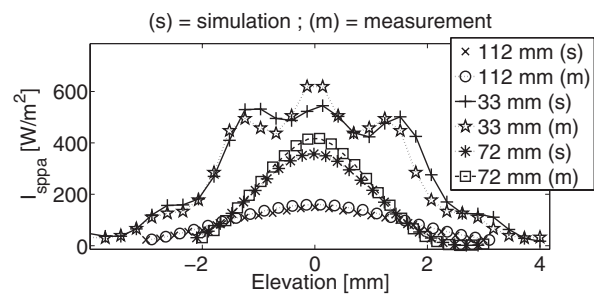

FIG. 9. Elevation intensity comparison between simulated and measured values at three depths. 
TABLE V. rms error at three depths with $1.7 \mathrm{~dB}$ amplitude correction.

\begin{tabular}{llrrlrc}
\hline \hline & \multicolumn{3}{c}{ rms \% intensity } & \multicolumn{3}{c}{ rms \% pressure } \\
\hline Depth (mm) & 33 & 72 & 112 & 33 & 72 & 112 \\
Element 64 & 11.5 & 14.2 & 5.9 & 26.2 & 26.3 & 27.2 \\
Element 65 & 19.5 & 16.2 & 14.6 & 25.2 & 27.1 & 26.7 \\
Element 66 & 15.2 & 7.1 & 5.8 & 26.0 & 26.2 & 26.1 \\
\hline \hline
\end{tabular}

through the whole elevation plane in the three depths 33,72 , and $112 \mathrm{~mm}$. However, it is pointed out that the $1.7 \mathrm{~dB}$ amplitude adjustment on the pressure pulses is also used here.

The consistent tendency on the shape of the intensity curves is due to the setup of the FIELD II program and is not to be credited the impulse response calculated by the transducer model. This is because the shape of the intensity profile is entirely dependent on the spatial impulse response. It is therefore to be concluded that the FIELD II setup with the many subdivided elements is performed properly.

A quantitative comparison between the simulated and measured intensity and pressure is shown in Table V. The table shows the relative rms error between the simulated and the measured pressure in percent, which is calculated similarly as in Eq. (40). The calculation is made using the measured pressure in the three different measurement points along the element's center lines. The center lines are found by searching for the maximum amplitudes of the measurements along the lateral direction. Accordingly the intensity rms error is calculated for the intensity studies in the elevation plane using the lateral average. Both results are listed in Table V.

From Table V, it is found that the pressure prediction at the three depths in between differ by $2 \% \mathrm{rms}$ error relative to the mean, which is a much better result than the one found for the piezoceramics in Table III. This indicates that the transducer is very homogeneous in its performance, and that the measurements along the center lines are performed uniformly. A comparison between simulated- and measured pressures corresponds to an rms error equal to $25.2 \%$ to $27.2 \%$. The rms intensity errors of the three elements in between are found to differ by $5.1 \%-14.6 \%$ at $112 \mathrm{~mm}, 7.1 \%-$ $16.2 \%$ at $72 \mathrm{~mm}$, and $11.5 \%-19.5 \%$ at $33 \mathrm{~mm}$. This increase in difference the closer the measurements are to the transducer is mainly due to the transducer's nonideal surface. Small deviations in the surface profile are of influence close to the transducer surface, since these are not accounted for in the FIELD II setup.

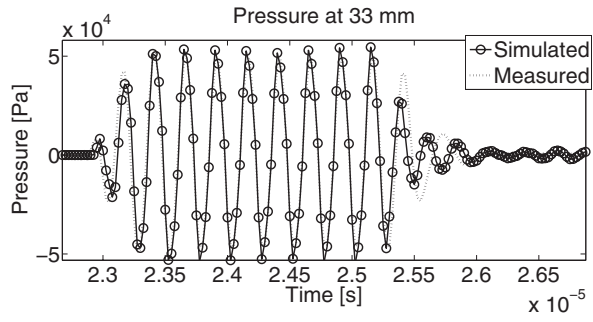

FIG. 10. Comparison between a simulated and a measured pressure pulse at a distance of $33 \mathrm{~mm}$.

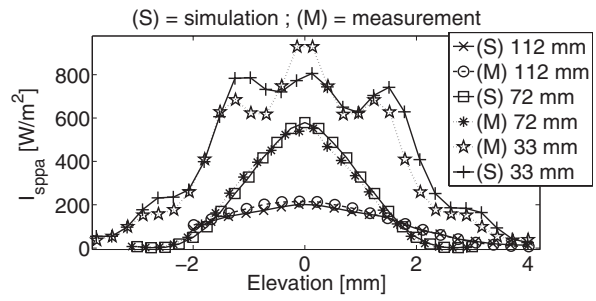

FIG. 11. Elevation intensity comparison between simulated and measured values at three depths using major elements only.

\section{The results on the convex transducer modeling only the major elements}

Figure 10 shows a comparison between simulated and measured pressures at a distance of approximately $33 \mathrm{~mm}$ from an emitting transducer element. The comparison does not involve an amplitude correction as seen in the previous results, which indicates that modeling of the major elements instead of each subelement predicts a better amplitude result. The same pulse behavior is, however, found with lacking information on pulse tails and slight overshoot on transitions.

Figure 11 is an intensity plot similar to Fig. 9. From the figure it can be identified that the amplitudes are well predicted and that the FIELD II surface model is predicting the intensity profile nicely in this configuration. The prediction is close to the one found in Fig. 9.

Table VI shows the quantitative rms errors for the intensity. As can be identified from the numerical errors on the pressure predictions only a small increase in the errors is found. Notice, that the data are given without amplitude correction which was not found necessary when using the given surface model of FIELD II. This indicates that the extra area contribution from the kerfs of the subdivided elements in between gives the extra small amplitude contribution needed for having a calibrated model. The intensity errors found in Table VI are found to be almost identical with those in Table $\mathrm{V}$, which also implies that the two transducer models are working almost identically.

\section{DISCUSSION}

Figure 5 shows that the free oscillating pulse tail may approach an almost $\pi / 2$ phase shift. Such a phase drift is due to several consequences of the presented modeling. One consequence is the use of a one-dimensional model which restricts the impulse response to account for dispersion modes that are only valid in the thickness mode (vibrations and dependencies along the $z$ coordinate only). The attenuation in this direction is, furthermore, modeled as being frequency independent. A more detailed model involves frequencydependent attenuation algorithms and a multidimensional

TABLE VI. rms error at three depths with $0 \mathrm{~dB}$ amplitude correction.

\begin{tabular}{llccccc}
\hline \hline & \multicolumn{3}{c}{ rms \% intensity } & \multicolumn{3}{c}{ rms \% pressure } \\
\hline Depth (mm) & 33 & 72 & 112 & 33 & 72 & 112 \\
Element 64 & 14.6 & 6.7 & 13.0 & 29.9 & 33.3 & 31.2 \\
Element 65 & 19.9 & 9.6 & 5.76 & 29.4 & 34.0 & 30.7 \\
Element 66 & 19.8 & 8.3 & 6.24 & 29.6 & 33.7 & 30.8 \\
\hline \hline
\end{tabular}


analysis. The amplitudes calculated in water may also be adjusted slightly since attenuation in water was not accounted for in FIELD II. This amounts to a maximum multiplication factor for the $112 \mathrm{~mm} 4 \mathrm{MHz}$ pressure pulse of approximately 0.96 which will yield an amplitude difference of approximately $0.3 \mathrm{~dB}$. Also inaccuracies of the input parameters are possible error sources. ${ }^{20}$ The simulations in this work rely on the exactness of the input parameters obtained by consulting the manufacturers and the work by Algueró et $a l .{ }^{13}$ as wells as the simplified electronic circuit. ${ }^{20}$ However, these parameters may be slightly different from those characterizing the samples used in the presented experiments. A correction of the free resonance frequency of the different transducers requires a fine tuning of especially the density $\rho$, the stiffness $c^{D}$, and the thickness $L$, by performing measurements on each individual transducer. For the pz27 samples also exact consideration of wire connections, soldering, and immersion medium would improve the errors, which to some extend is also applicable for the commercial transducer.

Despite the relatively high quantitative error found in this work it must also be argued that the model's simplicity makes it a qualified tool for fast hybrid modeling with FIELD II compared to hybrid modeling linked up against timeconsuming finite-element programs. Impulse responses, nevertheless, calculated using the latter programs benefit in the level of detail from the full dimensionality, however, it is also more sensitive to the accuracy of input parameters.

\section{CONCLUSION}

It has been shown that a one-dimensional model for ultrasound multilayer transducers can be implemented to predict the required volt-to-surface acceleration converted impulse response needed by the FIELD II software to calculate the field pressure in front of piezoceramic disk samples as well as from a more complicated 128 element convex ultrasound transducer. The implemented model predicts the current flow through simple electrical circuits driving piezoceramic disks within an rms error of $8.6 \%-36 \%$ for long and short pulses, respectively, and within $11.2 \%$ to $36.2 \%$ for pressure pulses, respectively. All pressure amplitudes were found to give an approximately $2 \mathrm{~dB}$ overshoot in prediction. Both the real-valued data set and the complex-valued data set were found to exhibit almost identical results, which implies that the model works well with both types of parameter sets. Furthermore, it implies that it is possible to model losses in the piezoceramic using the mechanical quality factor or complex material parameter sets.

Pressure measurements carried out on the medical convex transducer and compared to model predictions yield rms errors of $26 \%-30 \%$ depending on the surface model used. Generally, the different pressure measurements were found to differ within $2 \%$. Comparison of measured and simulated intensities was found to give an error between $5.8 \%$ and $19.8 \%$, where the largest errors were found close to the elements. The results also show that the simplified electronic circuit describing the transmitter unit and the coaxial cable models the voltage amplitude across the piezoelectric trans- ducer elements properly. It has been shown that using a measurement of the transmitter output voltage as an input excitation waveform for the FIELD II model gives good agreement between simulated and measured results. It can also be concluded that modeling of each single subdivided element gives almost the same result for the pulse shape as modeling the entire element as a whole surface does. However, an undershoot of approximately $1.7 \mathrm{~dB}$ was found.

The FIELD II program combined with the one-dimensional modeling principle is therefore a good candidate for performing fast hybrid modeling of acoustic fields.

\section{ACKNOWLEDGMENTS}

The authors would like to thank Sound Technology, Inc. for supplying the transducer information as well as giving support in discussions. Also a great thank to BK Medical Aps for their assistance. Furthermore, the authors would like to thank Dr. Erling Ringgaard, Ferroperm Piezocermics A/S, Kvistgård, Denmark, for delivering the piezoceramic samples and supplying data sets as well as giving support in the discussions.

${ }^{1}$ J. A. Jensen, "Field: A program for simulating ultrasound systems," Med. Biol. Eng. Comput. 4, 351-353 (1996).

${ }^{2}$ J. A. Jensen and N. B. Svendsen, "Calculation of pressure fields from arbitrarily shaped, apodized, and excited ultrasound transducers," IEEE Trans. Ultrason. Ferroelectr. Freq. Control 39, 262-267 (1992).

${ }^{3}$ M. Redwood, "Transient performance of a piezoelectric transducer," J. Acoust. Soc. Am. 33, 527-536 (1961).

${ }^{4}$ E. K. Sittig, "Transmission parameters of thickness-driven piezoelectric transducers arranged in multilayer configurations," IEEE Trans. Sonics Ultrason. SU-14, 167-174 (1967).

${ }^{5}$ R. Krimholtz, D. Leedom, and G. Matthaci, "New equivalent circuits for elementary piezoelectric transducers," Electron. Lett. 6, 398-399 (1970).

${ }^{6}$ T. R. Meeker, "Thickness mode piezoelectric transducers," Ultrasonics 10, 26-36 (1972).

${ }^{7}$ P. Marchal, F. Levarssort, L.-P. Tran-Huu-Hue, and M. Lethiecq, "Effects of acoustical properties of a lens on the pulse-echo response of a single element transducer," IEEE International Ultrasonics, Ferroelectrics, and Frequency Control Joint 50th Anniversary Conference, pp. 1651-1654 (2004).

${ }^{8}$ P. Maréchal, F. Levarssort, L.-P. Tran-Huu-Hue, and M. Lethiecq, "Lensfocused transducer modeling using an extended KLM model," Ultrasonics 46, 155-167 (2007).

${ }^{9} \mathrm{M}$. Willatzen, "Ultrasound transducer modeling-Received voltage signals and the use of half-wavelength window layers with acoustic coupling layers," IEEE Trans. Ultrason. Ferroelectr. Freq. Control 46, 1164-1174 (1999).

${ }^{10} \mathrm{M}$. Willatzen, "Ultrasound transducer modeling-General theory and applications to ultrasound reciprocal systems," IEEE Trans. Ultrason. Ferroelectr. Freq. Control 48, 100-112 (2001).

${ }^{11} \mathrm{D}$. Bæk, J. A. Jensen, and M. Willatzen, "Testing of a one dimensional model for Field II calibration," Proc.-IEEE Ultrason. Symp. , 1417-1420 (2008).

${ }^{12}$ D. Bæk, J. A. Jensen, and M. Willatzen, "Calibration of Field II using a convex ultrasound transducer," in Proceedings of the International Congress on Ultrasonics, Vol. 3, Issue 1 (2009).

${ }^{13} \mathrm{M}$. Algueró, C. Alemany, and L. Pardo, "Method for obtaining the full set of linear electric, mechanical and electromechanical coefficients and all related losses of a piezoelectric ceramic," J. Am. Ceram. Soc. 87, 209-215 (2004).

${ }^{14}$ B. A. Auld, Acoustic Fields and Waves in Solids, 2nd ed. (Krieger, Malabar, FL, 1990), Vol. 1.

${ }^{15}$ T. Tsurumi, H. Kakemoto, and S. Wada, "Dielectric, elastic and piezoelctric losses of PZT ceramics in resonate state," in Proceedings of the 13th IEEE International Symposium on Applications of Ferroelectrics, ISAF 2002 (2002), pp. 375-378.

${ }^{16}$ M. Castillo, P. Acevedo, and E. Moreno, "KLM model for lossy piezo- 
electric transducers," Ultrasonics 41, 671-679 (2003).

${ }^{17}$ J. G. Smits, "Iterative method for accurate determination of the real and imaginary parts of the materials coefficients of piezoelectric ceramics," IEEE Trans. Sonics Ultrason. 23-6, 393-401 (1976).

${ }^{18}$ C. Alemany, L. Pardo, B. Jimnez, F. Carmona, and J. Mendiola, "Automatic iterative evaluation of complex material constants in piezoelectric ceramics," J. Phys. D: Appl. Phys. 27, 148-155 (1994).
${ }^{19}$ J. A. Jensen, O. Holm, L. J. Jensen, H. Bendsen, H. M. Pedersen, K. Salomonsen, J. Hansen, and S. Nikolov, "Experimental ultrasound system for real-time synthetic imaging," Proc.-IEEE Ultrason. Symp. 2, 15951599 (1999).

${ }^{20} \mathrm{D}$. Bæk, M. Willatzen, and J. A. Jensen, "Parameter sensitivity study of a Field II multilayer transducer model on a convex transducer," Proc.-IEEE Ultrason. Symp. In press. 